
\title{
R Research Square \\ The mechanism of jian-Gan-Xiao-Zhi decoction in IR adipocytes and its component analysis
}

\section{Fang Fang}

Nanjing University Medical School

WeiBo Wen ( $\nabla$ WeiBoWen15@163.com )

Nanjing University Medical School

\section{Xuehua Xie}

Nanjing University Medical School

Ling Yang

Yunnan University of Traditional Chinese Medicine

\section{Xu Zhang}

First People's Hospital of Yunnan

Jie Zhao

First People's Hospital of Yunnan

\section{Research}

Keywords: Jian-Gan-Xiao-Zhi decoction, insulin resistant, ROS, JNK, AMPK, LC-MS/MS

Posted Date: March 24th, 2020

DOI: https://doi.org/10.21203/rs.3.rs-18749/v1

License: (c) (i) This work is licensed under a Creative Commons Attribution 4.0 International License.

Read Full License 


\section{Abstract}

Background : Jian-Gan-Xiao-Zhi decoction (JGXZ) is a formula of TCM and used to treat patients with NAFLD. Thus, the study aimed to analyze the mechanism of drug serum of JGXZ in adipocytes and detect the main components of JGXF in rat serum.

Methods : The 3T3-L1 preadipocytes were used to establish IR adipocytes model. The lipid accumulation in adipocytes was detected by oil red $O$ staining. After JGXZ treatment, glucose consumption, TC and TG were analyzed by corresponding kits. ROS levels were measured by flow cytometry. In addition, Westen Blot was used to assess LKB1/AMPK and JNK/IRS/PI3k/AKT expressions. Except for that, the main components of JGXF in rat serum samples were detected by LC-MS/MS method. A Phenomenex Luna C18 was used to perform chromatographic separation. The ion source is ESI. The mobile phase contained methanol and $0.1 \%$ formic acid solution.

Results: The results showed that JGXZ significantly decreased glucose levels and adipogenesis, accompanied by decreased IR. Besides, JGXZ markedly affected ROS, LKB1/AMPK and JNK/IRS/PI3K/AKT levels. The R1, Rg1, paeoniflorin, Rb1, astragaloside and tanshinone could be significantly quantified.

Conclusion: Thus, JGXZ decreased glucose and lipid synthesis possibly via ROS/AMPK/JNK pathway and R1, Rg1, paeoniflorin, Rb1, astragaloside and tanshinone in JGXZ played main roles in JGXZ treating NAFLD.

\section{Background}

Non-alcoholic fatty liver disease (NAFLD) was characterized by seatosis and adipose storage of liver parenchyma cells, which was generally believed to be closely related to IR. Improvement of IR was one of the important therapeutic measures for NAFLD. Insulin sensitizers including thiazolidinediones induced glucose uptake and lipid synthesis, which increased weight. Thus, the drug without inducing lipid synthesis has been desired. Adipose tissue is the main storage site for excess energy in the form of triglycerides, which provides up to $70 \%$ free fatty acids for synthesis of triglycerides in liver[1]. Thus, the adipose tissue impairment caused excess fatty acid, which was characterized by ectopic excessive lipid deposition, systemic insulin resistance and inflammation. More fatty acids were transported from adipose tissue to the triglyceride pool in the liver than were generated from DE novo synthesis of lipid in liver in NAFLD[2]. Lipid mobilization was strongly inhibited by insulin under normal physiological conditions. However, with the expansion of fat storage in the liver. Free fatty acids were increased and ameliorated the insulin signal, which accelerated IR. Adipose tissue was becoming a kind of mediator in nonalcoholic fatty liver disease and metabolic disease[3], and was a vital target organ for insulin which stimulates glucose uptake and inhibits lipid lipolysis. IR was closely correlated with liver damage in NAFLD $[4,5]$. Thus, it was an efficient way to recover the sensitivities of adipose to insulin for alleviating lipid toxicity of the liver. 
Liver kinase B1 (LKB1) was involved in inhibiting lipid synthesis and promoting glucose uptake via phosphorylating Thr-172 of AMPK which inactivated Acetyl-CoA Carboxylase and regulating 3-hydroxy-3methyl glutaryl coenzyme A reductas[6]. The study has suggested that AMPK activation could improve metabolic imbalances caused by type $\otimes$ diabetes[7]. ROS could activate JNK pathway via various pathways, the activation of which further phophorylated serine307 of IRS- 1 to inhibit the transduction of insulin signal [8]. Furthermore, the phosphorylation of serine307 of IRS-1 was the main mechanism in IR of body. Besides, insulin receptor substrate (IRS) could be phosphorylated by insulin receptor tyrosine kinase to mediate the transmission of insulin signal. Then, p-IRS bound to the $\mathrm{SH} 2$ domain of PI3K regulatory subunit p85, which recruited and activated PI3K. PI3K mediated the AKT activation. P-AKT could regulate glycogen synthesis, gluconeogenesis and glucose absorption through phophorylating corresponding substrates. Thus, ROS/JNK/IRS/PI3K/AKT affected the the conversion of glucose to glycogen and protein, and absorption.

Jian-Gan-Xiao-Zhi decoction (JGXZ) is a a formula of Traditional Chinese medicine (TCM) and could treat patients with non-alcoholic fatty liver disease. Previous animal experiment has shown that JGXZ decreased IR and affected AMPK/JNK levels of liver in non-alcoholic fatty liver rat model[9]. TCM was made up of many kinds of substances, which was broadly used to treat patients in China. However, some substances might be not effective for treating patients and the uncertainty of the active ingredients greatly limited its popularity. Besides, pharmacokinetic properties in TCM was usually poor. The long-term or overdose use of some TCM could cause certain toxicity reactions, which limited its clinical utilization to some extent[10].

Liquid chromatography-tandem mass spectrometry (LC-MS/MS) method has been developed for qualitative and quantitative analysis of components in traditional Chinese medicine including some components of lower content or not easily being separated[11, 12]. Therefore, it's important to determine the concentration of main components in serum after TCM administration for further analyzing active components and studying their mechanism. The study aimed to investigate the potential mechanism in of JGXZ in adipocytes and provides the theoretical support for the clinical application of JGXZ. In addition, the concentration of main components in serum after TCM administration was detected by LCMS/MS method.

\section{Materials And Methods}

\section{Preparation of serum containing jiangan xiaozhi decoction}

Sprague-Dawley(SD) rats were purchased from SPF(Beijing) Biotechnology (Beijing, China), which were $5 \sim 6$ weeks old and whose weight was $180-200 \mathrm{~kg}$. The experiment was approved by animal ethics committee of Yunnan University of TCM. Jian gan xiaozhi fang(JGXF) is consisted of Danshen $15 \mathrm{~g}$, notoginseng powder $6 \mathrm{~g}$, zedoary rhizome $15 \mathrm{~g}$, hawthorn $20 \mathrm{~g}$, astragalus $20 \mathrm{~g}$, qingpi $10 \mathrm{~g}$, red peony root $20 \mathrm{~g}$, turmeric $12 \mathrm{~g}$ alisma $15 \mathrm{~g}$, chrysanthemum $15 \mathrm{~g}$, lotus leaf $15 \mathrm{~g}$, liquorice $6 \mathrm{~g}$. SD rats were randomly divided into three groups(10 in each group, half male and female) , namely, Jiangan xiaozhi group 
$(21.73 \mathrm{~g} / \mathrm{kg})$, Metformin group $(0.2 \mathrm{mg} / \mathrm{kg})$ and normal saline control group $(5 \mathrm{ml} 0.9 \% \mathrm{Nacl})$. The rats were administered orally two times for three days. One hour after the last dose, the blood was collected from abdominal aorta and centrifuged at $3000 \mathrm{r} / \mathrm{min}$ for $15 \mathrm{~min}$ to collect serum. Afterwards, the serum was inactivated at $56^{\circ} \mathrm{C}$ for $30 \mathrm{~min}$ and filtered to eliminate bacteria using $0.22 \mu \mathrm{m}$ microfiltration membrane. HPLC detected the main content in Jiangan xiaozhi containing curcumin, notoginseng, astragaloside, paeoniflorin, tanshinone and so on. After passing inspection, the serum was put into $-80^{\circ} \mathrm{C}$ refrigerator to further experiment. The study was supported according to the National Institutes of Health Regulations and approved by the Institutional Animal Care and Use Committee at Yunnan University of TCM.

\section{Cells}

3T3-L1 preadipocytes were purchased by Chinese Academy of Sciences Shanghai cell bank. The cells were cultured in DMEM high sugar medium containing $10 \%$ fetal Bovine Serum, and $1 \%$ Penicillin and streptomycin at $37^{\circ} \mathrm{C}, 5 \% \mathrm{CO} 2$. After cell adherence, the medium was replaced. The cells of logarithmic phase were inoculated on the culture plate, which then were induced differentiation $48 \mathrm{~h}$ after cell contact inhibition. The medium containing $0.5 \mathrm{mmol} / \mathrm{I} \mathrm{IBXM}, 1.0 \mu \mathrm{mol} / \mathrm{L}$ DEX, $10 \mu \mathrm{g} / \mathrm{ml}$ INS replaced original medium and continued to culture cells for 48 hours. Then, $10 \mu \mathrm{g} / \mathrm{ml}$ INS medium continued to culture cells for $48 \mathrm{~h}$. the medium was replaced by DMEM and observed $7 \mathrm{~d}$. After that, oil red 0 was used to stain fat in cells and determine whether cells were differentiated and mature (sigma, America). The cells were used for further study when more than $90 \%$ cells presented "ring" lipid droplet. The mature adipocytes were divided into control and model group. The control group was cultured in DMEM medium. The model group was provided with the medium containing $1 \mu \mathrm{mol} / \mathrm{L}$ DEX to establish IR adipocyte model. $48 \mathrm{~h}$ after JGXZ or Rosiglitazone treatment, Glucose content kit (YanJin, Shanghai, China) was used to detect glucose levels in medium for evaluating insulin sensitivity of cells. The absorbance at $505 \mathrm{~nm}$ was detected. The absorbance in blank, standard and measured tube were recorded as A1, A2 and A3 respectively. The glucose contents were calculated by the formula: glucose contents $(\mu \mathrm{mol} / \mathrm{mg}$ prot $)=(C$ standard $\times \mathrm{V} 1) \times(\mathrm{A} 3-\mathrm{A} 1) \div(\mathrm{A} 2-\mathrm{A} 1) \div(\mathrm{V} 1 \times \mathrm{Cpr})=0.5 \times(\mathrm{A} 3-\mathrm{A} 1) \div(\mathrm{A} 2-\mathrm{A} 1) \div \mathrm{Cpr}(\mathrm{V} 1$ is sample volume, $\mathrm{Cpr}$ is protein concentrations, ,C standard is standard substance concentration).

\section{TC and TG contents}

After JGXZ or Rosiglitazone treatment, the cells were washed with PBS twice and broken by ultrasound under the condition of ice bath. Then, the prepared cell homogenate was used to detect TC levels by TC kits according to the protocol, along with the detection of TG contents via TG kits according to the protocol (NanJing JianCheng, China).

\section{The detection of ROS}

The cell suspension was prepared, the concentration of which was $5^{\star} 10^{4}$ cells/well. The cell was seeded into 6-well plate and induced differentiation. The cells suspension was prepared through digestion with pancreatin $48 \mathrm{~h}$ after JGXZ or Rosiglitazone treatment. Then, the cells were centrifuged at $1000 \mathrm{r} / \mathrm{min}$ for $5 \mathrm{~min}$. After using PBS to wash cells twice, the cell precipitate was collected by centrifugation. ROS Assay 
Stain Solution (eBioscience, America) of $100 \mu \mathrm{L}$ was added to each tube, the blank group without dye was set as the control. The green fluorescence intensity of DCFH-DA was detected by flow cytometry after incubation at $37^{\circ} \mathrm{C}$ for $60 \mathrm{~min}$.

\section{Western blot}

The cells were collected into the EP tube, which then was add protein lysate. After centrifuging for $10 \mathrm{~min}$ at $12000 \mathrm{r} / \mathrm{min}$ at $4^{\circ} \mathrm{C}$ for $15 \mathrm{~min}$. The supernatant was collected and the protein concentration was detected by BCA method. The target protein was separated by using SDS-PAGE electrophoresis. Blots was blocked in skimmed milk powder solution at $37^{\circ} \mathrm{C}$ for $2 \mathrm{~h}$. Afterwards, the blots were incubated with the primary antibody at $4^{\circ} \mathrm{C}$ overnight. The blots were washed and incubated with secondary antibody. The bands were analyzed colored with ECL solution.

\section{LC-MS/MS method for analyzing the components of JGXF}

The 9 main components of JGXZ was determined in LC-MS/MS instrument (Triple Quad ${ }^{\text {TM }}$ 5500, Applied Biosystem Sciex, America). Chromatographic separation was performed on a Phenomenex Luna C18 $(150 \mathrm{~mm} \times 2.0 \mathrm{~mm}, 3 \mu \mathrm{m})$, and conducted by the gradient program as shown in table1. The running time of each needle sample is $8 \mathrm{~min}$ and the column temperature was kept at $40^{\circ} \mathrm{C}$. $0-4 \mathrm{~min}$ belonged to the negative ion detection mode. Analytes were detected with the following mass spectrometer source settings. ESI source; ion spray voltage: $-4500 \mathrm{~V}$; temperature: $500^{\circ} \mathrm{C}$; collision gas $\left(\mathrm{N}_{2}\right)$ pressure :8psi; nebulizer gas $\left(\mathrm{N}_{2}\right)$ pressure: 50psi; heater gas $\left(\mathrm{N}_{2}\right)$ pressure:50psi; curtain gas $\left(\mathrm{N}_{2}\right)$ pressure: 35psi; inlet potential (EP): -10V; the ion parameters used for quantitative analysis are respectively: notoginsenoside R1 m/z 977.3 $\rightarrow$ 931.4, DP囚-134凹CE囚-42; Ginsenoside Rg1 m/z 845.3 DP: -166.3 CE: -44.3; Paeoniflorin m/z 525.1 $\rightarrow 449.2$ DP: -63.5 CE: -21.1; Internal standard naringin m/z 579.2 $\rightarrow 271$ DP: -214 CE: -44. The results of ion scan were shown in Figure4. 4-8min belonged to the positive ion detection mode. Analytes were detected with the following mass spectrometer source settings. ESI source; ion spray voltage: $5500 \mathrm{~V}$; temperature: $500^{\circ} \mathrm{C}$; collision gas $\left(\mathrm{N}_{2}\right)$ pressure :8psi; nebulizer gas $\left(\mathrm{N}_{2}\right)$ pressure: 50psi; heater gas $\left(\mathrm{N}_{2}\right)$ pressure: 35psi; curtain gas $\left(\mathrm{N}_{2}\right)$ pressure: 35psi; inlet potential (EP): 10V; the ion parameters used for

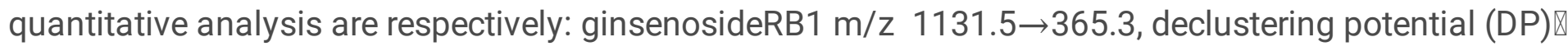
219.8, collision energy (CE) $₫ 76.9$ Astragaloside $\mathrm{m} / \mathrm{z}$ 785.6 $\rightarrow$ 143.1 DP:123.2 CE:16.8; Curcumin m/z $369.2 \rightarrow 285.2$, declustering potential (DP): 87, collision energy (CE):21.9; Entanshinone $\mathrm{m} / \mathrm{z} 297.2 \rightarrow 251.2$ DP:168 CE:32.2; Tanshinone m/z 277.1 $\rightarrow 249.1$ DP:191.5 CE:28.9; Tanshinone A m/z 295.2 $\rightarrow 277.2$ DP:174.7 CE:26.4; Internal standard cannabidiol m/z 315.2 $\rightarrow 193.1$ DP:90.6 CE:28.8. The results of ion scan were shown in Figure5.

\section{Preparation and quantitative analysis of serum containing drug in rats}

SD rats $(n=6$, half male and half female) were randomly divided into blank group $(n=5)$ and $\operatorname{JGXF}(n=1$, marked as 3 ) group. The blood was collected by eye socket. The serum of $100 \mu \mathrm{L}$ was placed into a blank centrifuge tube as blank serum group. After vortexed for 30 seconds, a mixture of methanol-acetonitrile $(200 \mu \mathrm{L})$ in a 1:1 ratio was added into the tube. When vortexed for 1 min and mixed well, the mixture was 
centrifuged for $5 \mathrm{~min}$ at $10000 \mathrm{r} / \mathrm{min}$ and the supernatent of $100 \mu \mathrm{L}$ was collected, among which $5 \mu \mathrm{L}$ was used to sample injection. Chromatographs were taken as shown in Figure 4. The mixture solution of reference substance $(10 \mu \mathrm{L}, 1000 \mathrm{ng} / \mathrm{ml})$ was added into blank serum of $90 \mu \mathrm{L}$. Then, sample injection of 5 $\mu \mathrm{L}$ was obtained through same way. The dose equivalent to $32 \mathrm{~g} / \mathrm{kg}$ of JGXF was given rats by gavage twice a day in interval of 12 hours for three consecutive days. Then, the blood was collected through the abdominal aorta $1 \mathrm{~h}$ after the last JGXT administration. The blood was stored at $4{ }^{\circ} \mathrm{C}$ overnight. Afterwards, the serum was collected through centrifuging at $3000 \mathrm{r} / \mathrm{min}$ for $15 \mathrm{~min}$ and inactivated at $56^{\circ} \mathrm{C}$ for $30 \mathrm{~min}$, then filtered and sterilized with microporous membrane of $0.22 \mu \mathrm{m}$. The serum of $100 \mu \mathrm{L}$ was placed into a blank centrifuge tube, followed by the addition of the mixed internal standard solution of $10 \mu \mathrm{L}$ (containing naringin of $10000 \mathrm{ng} / \mathrm{ml}$, cannabidiol of $1000 \mathrm{ng} / \mathrm{ml}$ ). Similarly, sample injection of 5 $\mu \mathrm{L}$ was obtained through same way.

\section{Quantitative range of nine compounds in serum}

The quantitative ranges of 9 compounds in serum were as follows: notoginsenoside R1:0.5 $50 \mathrm{ng} / \mathrm{mL}$; Ginsenoside Rg1:1 100 ng/mL;Paeoniflorin: 5 500 ng/mL; Ginsenoside Rb1:10 1000 ng/mL; Astragaloside : $0.1 \sim 10 \mathrm{ng} / \mathrm{mL}$;Curcumin: $1 \sim 100 \mathrm{ng} / \mathrm{mL}$; Cryptotanshinone:1 100ng/mL;

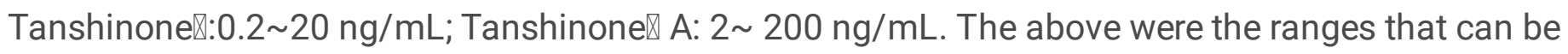
determined for nine compounds in serum.

\section{Determination of 9 main components in serum samples of JGXZ}

The dose equivalent to $32 \mathrm{~g} / \mathrm{kg}$ of JGXF was given rats ( $\mathrm{n}=12$, half male and female) by gavage twice a day in interval of 12 hours for three consecutive days. Then, the blood was collected through the abdominal aorta $1 \mathrm{~h}$ after the last JGXT administration.

\section{Statistical analysis}

The experimental data was analyzed using Graph Pad software. $P<0.05$ was considered as statistically significant.

\section{Results}

\section{T3-L1 IR adipocytes model}

The 3T3-L1 preadipocytes were long fusiform and presented no lipid droplet (LD). After cells were induced differentiation, the cells became larger and rounder with lipid droplets appearing in the cytoplasm. The small LDs appeared in the cytoplasmic, which were the reasons for the lipid beginning to accumulate. With the extension of time in cell culture, the small LDs would merge into larger LDs. Nucleus and organelles of mature adipocytes were squeezed to the side of cells by larger LD. Besides, cells were round. In 7 day, oil red 0 staining indicated that large amounts of LDs were stained red in cytoplasm, which formed "ring" structure around the nucleus. 95\% cells differentiated mature adipocytes according to counting cells randomly (Figure1) 


\section{JGXZ markedly reduced lipid deposition in IR adipocytes}

The lipid droplet was the organelle which was closely related to lipid storage, metabolism and secretion and consist of a single layer of phospholipid and a hydrophobic core composed of neutral lipids. The hydrophobic core mainly includes TG and cholesterol esters. The results showed that JGXZ significantly reduced the LDs levels (Figure2A). The glucose contents in the model group were significantly reduced comparing with control group. It replied that cells became insulin resistant(Figure2B). Furthermore, TG and TC were notably reduced by JGXZ(Fig2C-D). Thus, JGXZ significantly reduced lipid deposition in IR adipocytes. In addition, high-dose JGXZ has similar effects to Rosiglitazone in decreasing lipid deposition.

\section{JGXZ affected AMPK and ROS/JNK/IRS/PI3K/AKT levels}

After medicine treatment. LKB1 and AMPK were significantly increased by JGXZ or Rosiglitazone. It might reply that JGXZ affected lipid deposition and IR partly via LKB1/AMPK. Besides, JGXZ of low concentration significantly increased ROS production in IR adipocytes, the effects of which was similar to rosiglitazone treatment (Figure3A-B). However, ROS levels gradually recovered normal with the increase of the JGXZ concentration compared with control group. However, the effects of JGXZ with high concentration on AMPK/JNK/IRS/PI3k/AKT levels were similar to rosiglitazone treatment in IR adipocytes (Fig3C). Moreover, the phosphorylation levels of the Ser-473 and Thr-308 residues were all significantly increased after JGXZ treatment. The complexity JGXZ components might explain this phenomenon. Therefore, the results might indicate that JGXZ reduced lipid deposition and IR partly through ROS/AMPK/JNK pathway.

\section{Content of main contents in JGXZ in rat serum}

LC-MS/MS method was used to determine and quantify nine main ingredients in JGXZ in rat serum. After JGXZ administration in rats for consecutive three days. The concentrations of R1, Rg1, paeoniflorin, $\mathrm{Rb} 1$, astragaloside and tanshinone were quantified by LC-MS/MS method as shown in Table2. Besides, the paeoniflorin and Rb1 have higher concentrations compared with other components of JGXZ.

\section{Chromatograms of nine analytes}

The chromatograms of nine analytes were showed in Figure 4 and 5 . The blank serum in the blank group was used to perform chromatographic analysis under anion detection as shown in Figure 4A. The chromatograms of serun containng nine analytes and JGXZ were separately shown in Figure 4B and C. The blank serum in the blank group was used to perform chromatographic analysis under cationic mode as shown in Figure 5A. The chromatograms of serun containng nine analytes and JGXZ were separately shown in Figure 5B and C.

\section{Content of nine components in JGXZ}


Since No. 5 rat died after the last administration, the blood wasn't collected. The concentration of nine main compounds in serum containing JGXZ was determined (Table2). According to the identification of the nine components in serum containing JGXZ in rats, Rb was most abundant. However, the concentrations of curcumin, cryptotanshinone and tanshinone weren't significantly detected by LCMS/MS.

\section{Discussion}

Although the study has suggested that ROS in mitochondrial could not contribute to IR in adipocytes[13], there has a study shown that ROS inhibitors reduced IR caused by chronic insulin treatment[14]. In our study, JGXZ of high dose significantly reduced ROS levels, accompanied by decreased lipid disposition in adipocyte, the effects of which was reversible to Rosiglitazone. It indicated that except for decreasing IR and increasing glucose uptake, JGXZ also could decrease lipid synthesis, which was mediated by decreasing ROS levels. Thus, the complexity of JGXZ components was main reason. The study has suggested that ROS promoted lipid disposition in liver[15]. Furthermore, ROS was the main reason of IR in adipocytes. Besides, the studies have shown that reduced ROS was closely related with decreased lipid accumulation and the suppression of ROS in mitochondrial meditated preadipocytes differentiation[1618].

ROS was reported to involve in regulating insulin signally pathway and induce JNK activation[14]. However, with the increased concentration of JGXZ, ROS presented reversal trend with p-JNK levels in our study, which suggested that JNK pathway was also activated by other molecular apart from ROS.

ROS was not only involved in promoting AMPK phosphorylation, but also ROS as upstream signals was involved in the activation of AMPK[19, 20]. Besides, redox status modulated AMPK effects and antioxidant reduced lipid accumulation via activating AMPK in adipocyte[21]. However, the trend of AMPK was different from ROS after JGXZ treatment. Thus, JGXZ activated AMPK possibly not only through ROS mediation. More and more studies showed that AMPK activation could decreased lipid accumulation in adipocytes[16, 22]. JGXZ reduced lipid accumulation possibly partly via LKB1/AMPK pathway. The studies has suggested that AMPK activation could phosphorylate ACC, and inhibit fatty acids synthesis and promote fatty acids oxidation, which was the key enzyme of fatty acids synthesis[23, 24]. Besides, insulin signalling was significantly increased at IRS/AKT pathway by JGXZ. It t indicated that JGXZ recovered the sensitivities of adipocytes to insulin. In our study, JGXZ could significantly reduce DEX-induced IR in adipocytes and increased glucose uptake possibly through AMPK, ROS adn JNK/IRS/PI3k/AKT pathway.

To evaluated blood concentration of JGXZ in rats, LC-MS/MS method was used to detect the main components in serum. Then, the study found that the concentrations of R1, Rg1, paeoniflorin, Rb1, astragaloside and tanshinone in rat serum could be determined by LC-MS/MS method, which indicated that they played important roles in adipocyte IR induced by DEX. Thus, JGXZ exerted significant effects 
on decreasing glucose and lipid disposition possibly mainly through these components. The results were essential for JGXZ use in clinic and further studying the effects of these components in NAFLD.

\section{Conclusions}

JGXZ decreases glucose and lipid disposition via regulating ROS/ LKB1/AMPK and JNK/IRS/PI3K/AKT pathway. R1, Rg1, paeoniflorin, Rb1, astragaloside and tanshinone in JGXZ played main roles in JGXZ treating NAFLD, which is important for us to understand the mechanism of JGXZ in NAFLD.

\section{Abbreviations}

JGXZ: Jian-Gan-Xiao-Zhi decoction. NAFLD \Non-alcoholic fatty liver disease. LKB1『Liver kinase B1. IRS』 insulin receptor substrate. TCM: Traditional Chinese medicine. LC-MS/MS: Liquid chromatographytandem mass spectrometry. SD:Sprague-Dawley. LD: lipid droplet. AMPK:Adenosine 5'-monophosphate (AMP)-activated protein kinase. JNK: c-Jun N-terminal kinase. PI3k: phosphatidylinositol 3-kinase. AKT: PKB Protein Kinase

\section{Declarations}

\section{Acknowledgement}

This work was supported by the National Natural Science Foundation of China (The Study of Jiangan Xiaozhi Recipe Improving Non-alcoholic Fatty Liver Disease by Regulating Insulin Resistance on Basis of AMPK/ROS/JNK Signaling Pathway, 81560772).

\section{Conflict of interest}

The authors declare no conflict of interest.

\section{References}

[1] Barrows BR and Parks EJ. Contributions of different fatty acid sources to very low-density lipoprotein-triacylglycerol in the fasted and fed states. J Clin Endocrinol Metab 2006; 91: 1446-1452.

[2] Greenberg AS, Coleman RA, Kraemer FB, McManaman JL, Obin MS, Puri V, Yan QW, Miyoshi H and Mashek DG. The role of lipid droplets in metabolic disease in rodents and humans. J Clin Invest 2011; 121: $2102-2110$.

[3] Cusi K. Role of obesity and lipotoxicity in the development of nonalcoholic steatohepatitis: pathophysiology and clinical implications. Gastroenterology 2012; 142: 711-725.e716.

[4] Chen G and Goeddel DV. TNF-R1 signaling: a beautiful pathway. Science 2002; 296: 1634-1635. 
[5] Hotamisligil GS and Spiegelman BM. Tumor necrosis factor alpha: a key component of the obesitydiabetes link. Diabetes 1994; 43: 1271-1278.

[6] Wang F, Yang X, Lu Y, Li Z, Xu Y, Hu J, Liu J and Xiong W. The natural product antroalbol H promotes phosphorylation of liver kinase B1 (LKB1) at threonine 189 and thereby enhances cellular glucose uptake. J Biol Chem 2019; 294: 10415-10427.

[7] Zhang BB, Zhou G and Li C. AMPK: an emerging drug target for diabetes and the metabolic syndrome. Cell Metab 2009; 9: 407-416.

[8] Jaeschke A, Czech MP and Davis RJ. An essential role of the JIP1 scaffold protein for JNK activation in adipose tissue. Genes Dev 2004; 18: 1976-1980.

[9] Xue-Hua Xie J-BL, Fang Fang, Jie Zhao, Yong-Jun Cao, Huan-Tian Cui, Hong-Wu Wang, Zhai-Yi Zhang, Zhao-Hui Sun, Yuan Yin, Wei-Bo Wen. <span>Jian-Gan-Xiao-Zhi decoction ameliorates high-fat high-carbohydrate diet-induced non-alcoholic fatty liver disease and insulin resistance by regulating the AMPK/JNK pathway</span>. <span style='display:none' $>1</$ span>Traditional Medicine Research 1-12.

[10] Su T, Cheng BC, Fu XQ, Li T, Guo H, Cao HH, Kwan HY, Tse AK, Yu H, Cao H and Yu ZL. Comparison of the toxicities, activities and chemical profiles of raw and processed Xanthii Fructus. BMC Complement Altern Med 2016; 16: 24.

[11] Liang C, Yin J, Ma Y, Zhang X and Zhang L. Quantitative determination of characteristic components from compound of Lysionotus pauciflorus Maxim. by LC-MS/MS and its application to a pharmacokinetic study. J Pharm Biomed Anal 2020; 177: 112835.

[12] Jie Y, Mei-Zhen S, Jing W, Yun-Fei L, Pei L, Lin Q and Zhaorigetu B. In vitro cytotoxicity and in vivo acute and chronic toxicity of Xanthii Fructus and its processed product. BioMed research international 2013; 2013:

[13] Martin SD, Morrison S, Konstantopoulos N and McGee SL. Mitochondrial dysfunction has divergent, cell type-dependent effects on insulin action. Mol Metab 2014; 3: 408-418.

[14] Ge X, Yu Q, Qi W, Shi X and Zhai Q. Chronic insulin treatment causes insulin resistance in 3T3-L1 adipocytes through oxidative stress. Free Radic Res 2008; 42: 582-591.

[15] Jin Y, Tan Y, Chen L, Liu Y and Ren Z. Reactive Oxygen Species Induces Lipid Droplet Accumulation in HepG2 Cells by Increasing Perilipin 2 Expression. Int J Mol Sci 2018; 19:

[16] Liu M, Zheng M, Cai D, Xie J, Jin Z, Liu H and Liu J. Zeaxanthin promotes mitochondrial biogenesis and adipocyte browning via AMPKalpha1 activation. Food Funct 2019; 10: 2221-2233.

[17] Li G, Luan G, He Y, Tie F, Wang Z, Suo Y, Ma C and Wang H. Polyphenol Stilbenes from Fenugreek (Trigonella foenum-graecum L.) Seeds Improve Insulin Sensitivity and Mitochondrial Function in 3T3-L1 
Adipocytes. Oxid Med Cell Longev 2018; 2018: 7634362.

[18] Liu GS, Chan EC, Higuchi M, Dusting GJ and Jiang F. Redox mechanisms in regulation of adipocyte differentiation: beyond a general stress response. Cells 2012; 1: 976-993.

[19] Tian ZH, Weng JT, Shih LJ, Siao AC, Chan TY, Tsuei YW, Kuo YC, Wang TS and Kao YH. Arecoline inhibits the growth of 3T3-L1 preadipocytes via AMP-activated protein kinase and reactive oxygen species pathways. PLoS One 2018; 13: e0200508.

[20] Hwang JT, Kim SH, Lee MS, Kim SH, Yang HJ, Kim MJ, Kim HS, Ha J, Kim MS and Kwon DY. Antiobesity effects of ginsenoside Rh2 are associated with the activation of AMPK signaling pathway in 3T3L1 adipocyte. Biochem Biophys Res Commun 2007; 364: 1002-1008.

[21] Jager S, Handschin C, St-Pierre J and Spiegelman BM. AMP-activated protein kinase (AMPK) action in skeletal muscle via direct phosphorylation of PGC-1alpha. Proc Natl Acad Sci U S A 2007; 104: 12017-12022.

[22] Herranz-Lopez M, Barrajon-Catalan E, Segura-Carretero A, Menendez JA, Joven J and Micol V. Lemon verbena (Lippia citriodora) polyphenols alleviate obesity-related disturbances in hypertrophic adipocytes through AMPK-dependent mechanisms. Phytomedicine 2015; 22: 605-614.

[23] Peng IC, Chen Z, Sun W, Li YS, Marin TL, Hsu PH, Su MI, Cui X, Pan S, Lytle CY, Johnson DA, Blaeser F, Chatila T and Shyy JY. Glucagon regulates ACC activity in adipocytes through the CAMKKbeta/AMPK pathway. Am J Physiol Endocrinol Metab 2012; 302: E1560-1568.

[24] Qin Y, Yang RY, Chen MG, Shen XL and Ying-Jie HU. Inhibitory effect of cajanonic acid A on lipogenesis and lipolysis in 3T3-L1 adipocytes. Chinese Pharmacological Bulletin 2016;

\section{Tables}

Table I Chromatographic separation was conducted by the gradient program. 
time $\square \min \square \quad$ Flow rate: $\square \mathrm{mL} / \mathrm{min} \square \quad$ methanol $\quad 0.1 \% 0.1 \%$ formic acid

\begin{tabular}{llll}
\hline 0 & 0.5 & $20 \%$ & $80 \%$ \\
0.1 & 0.5 & $20 \%$ & $80 \%$ \\
2.5 & 0.5 & $75 \%$ & $25 \%$ \\
3 & 0.5 & $75 \%$ & $25 \%$ \\
3.5 & 0.5 & $100 \%$ & $0 \%$ \\
5 & 0.5 & $100 \%$ & $0 \%$ \\
5.1 & 0.5 & $20 \%$ & $80 \%$ \\
8 & 0.5 & $20 \%$ & $80 \%$ \\
\hline
\end{tabular}

Table II Connetrations of 9 main contents in JGXZ in rat serum were detected by LC-MS/MS. (n=12, No. 5 rat died).

\begin{tabular}{|c|c|c|c|c|c|c|c|c|c|}
\hline number & $\mathrm{R} 1$ & Rg1 & paeoniflorin & $\mathrm{Rb} 1$ & astragaloside & curcumin & cryptotanshinone & tanshinone $\llbracket \mathrm{A}$ & tanshinone[ \\
\hline 1 & 1.77 & 0.556 & 45 & 144 & 0.344 & ND & ND & 0.211 & ND \\
\hline 2 & 55.7 & 38.9 & 223 & 635 & 5.49 & ND & ND & 0.229 & ND \\
\hline 3 & 2.6 & 1.41 & 85.8 & 153 & 0.446 & ND & ND & 0.11 & ND \\
\hline 4 & 9.41 & 10.8 & 145 & 161 & 0.591 & ND & ND & 0.157 & ND \\
\hline 6 & 2.53 & 1.89 & 94.3 & 202 & 0.663 & ND & ND & 0.219 & ND \\
\hline 7 & 3.26 & 4.18 & 112 & 261 & 0.466 & ND & ND & 0.184 & ND \\
\hline 8 & 1.02 & 1.4 & 63.6 & 152 & 0.403 & ND & ND & 0.147 & ND \\
\hline 9 & 7.74 & 9.42 & 368 & 358 & 0.928 & ND & ND & 0.154 & ND \\
\hline 10 & 4 & 7.33 & 161 & 193 & 0.61 & ND & ND & 0.183 & ND \\
\hline 11 & 0.967 & 2.14 & 60.9 & 132 & 0.223 & ND & ND & 0.113 & ND \\
\hline 12 & 1.56 & 2.93 & 51.7 & 126 & 0.354 & ND & ND & 0.15 & ND \\
\hline
\end{tabular}

\section{Figures}




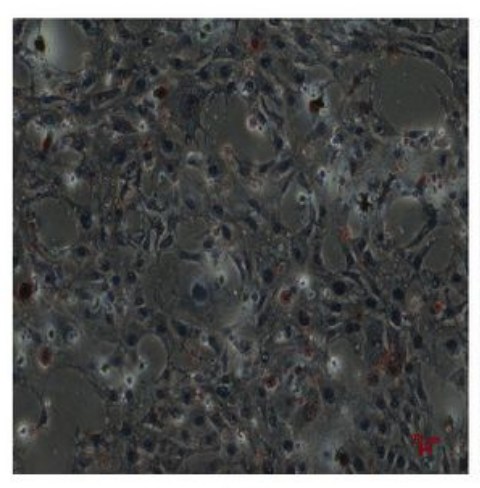

$1 d$

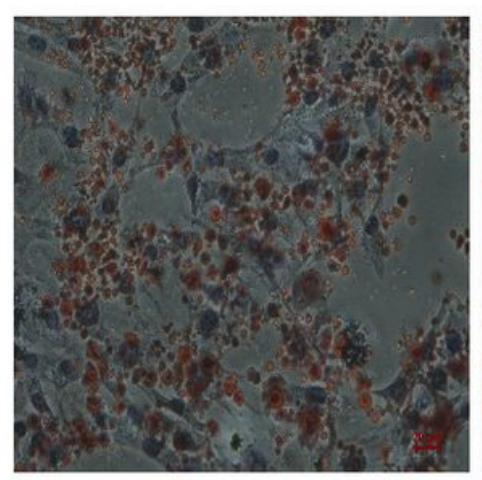

$5 d$

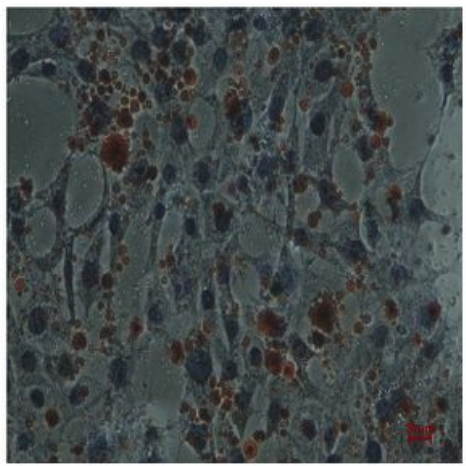

$2 d$

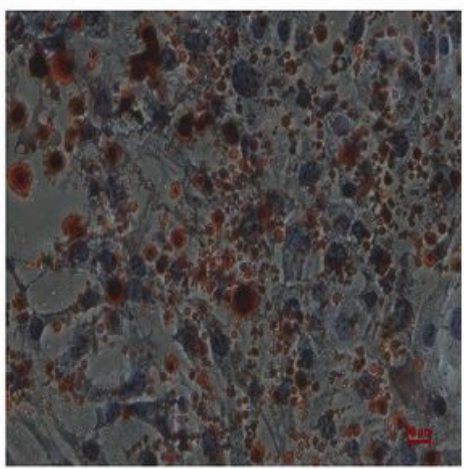

$6 d$

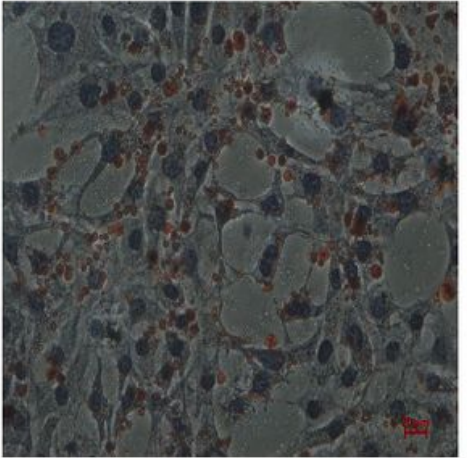

$3 d$

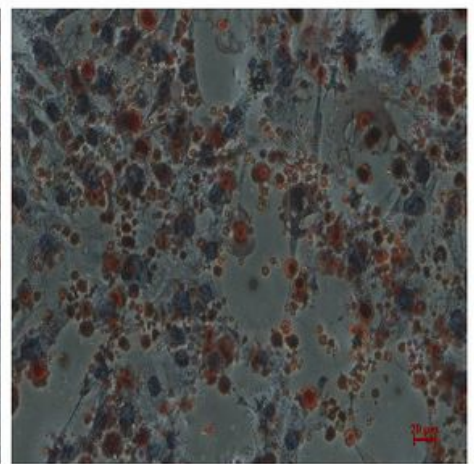

$7 d$

Figure 1

Oil red staining showed preadipocytes differentiation. 


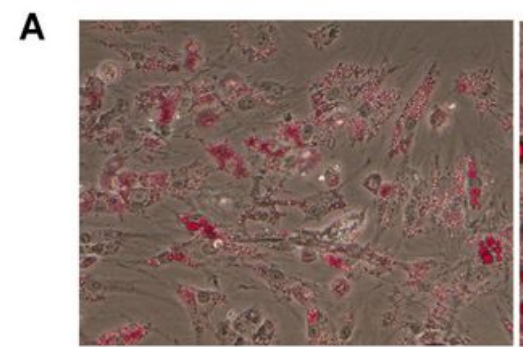

Control

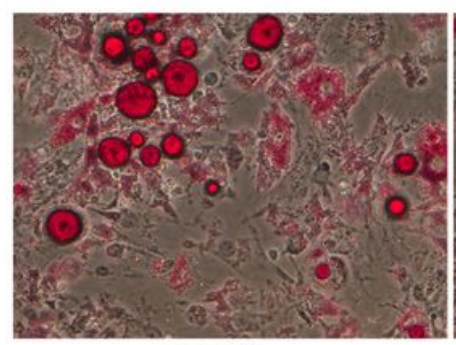

JGXZ-low

B

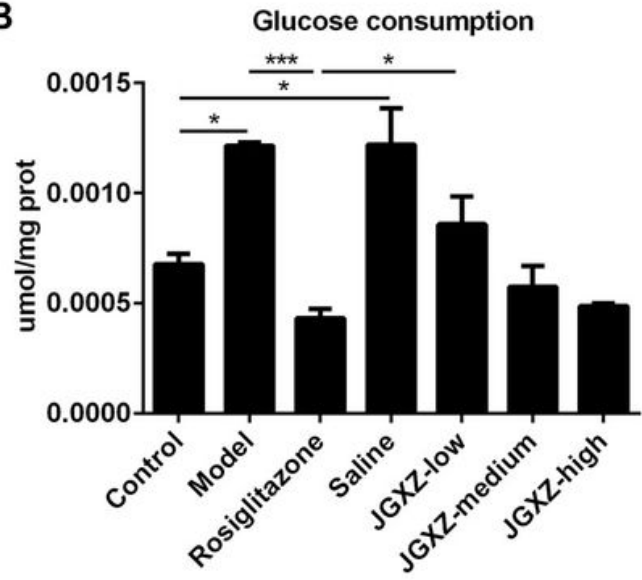

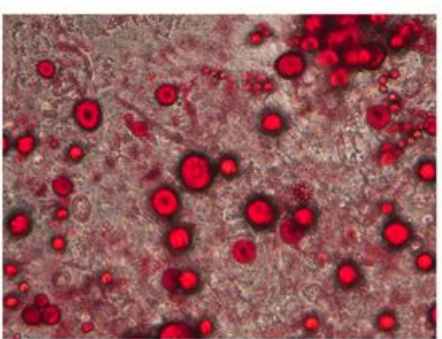

Model

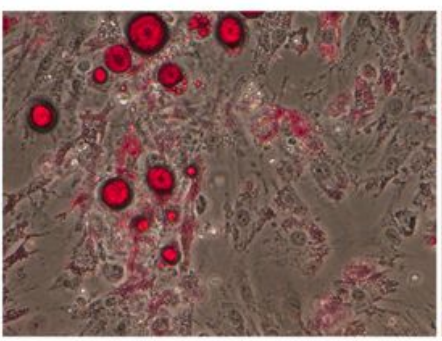

JGXZ-medium

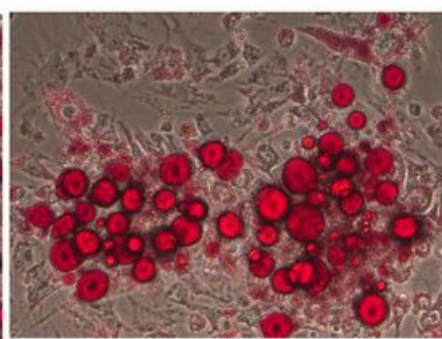

Rosiglitazone

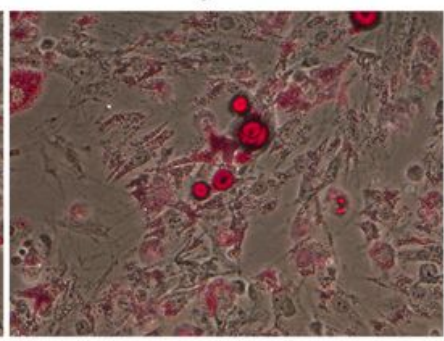

JGXZ-high
C

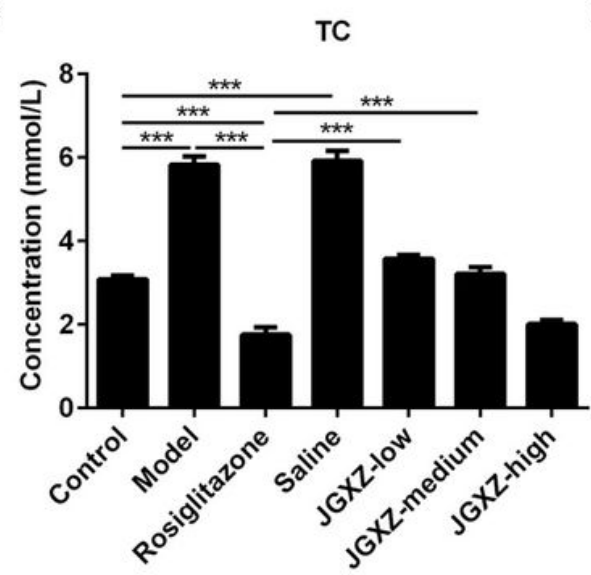

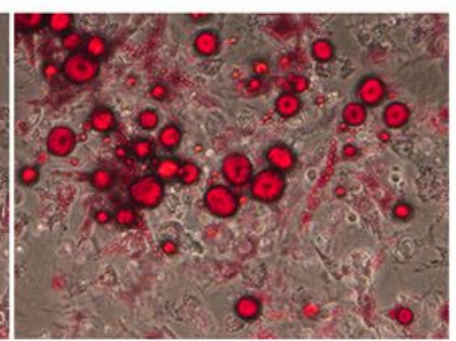

Saline

D

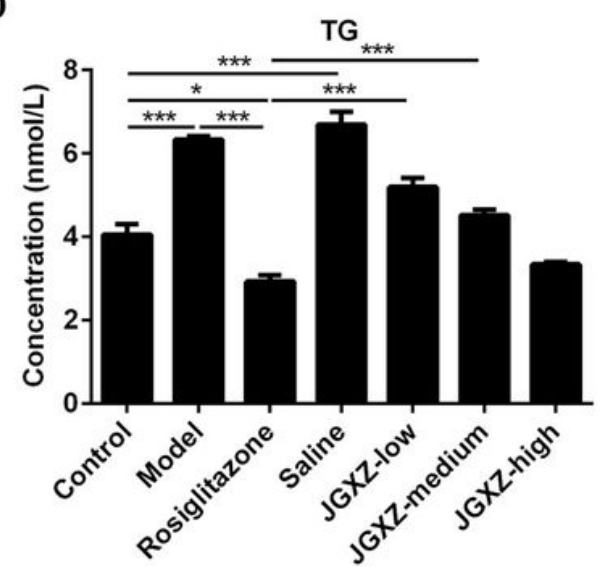

Figure 2

A the effects of serum containing drug on lipid accumulation for IR adipocytes. B the effects of serum containing drug on glucose consumption for IR adipocytes. $C$ the effects of serum containing drug on TC for IR adipocytes. D the effects of serum containing drug on TG for IR adipocytes. ${ }^{*} \mathrm{p}<0.05$ 

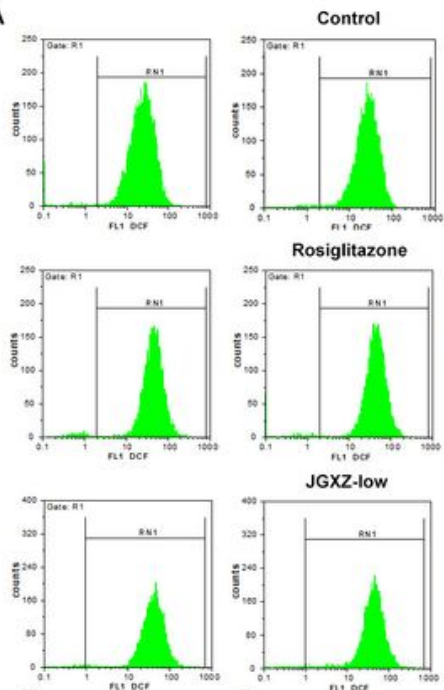

JGXZ-high
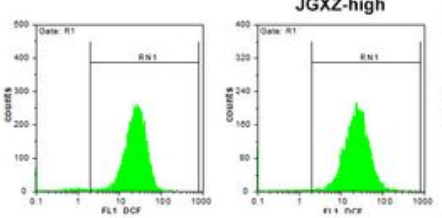

B
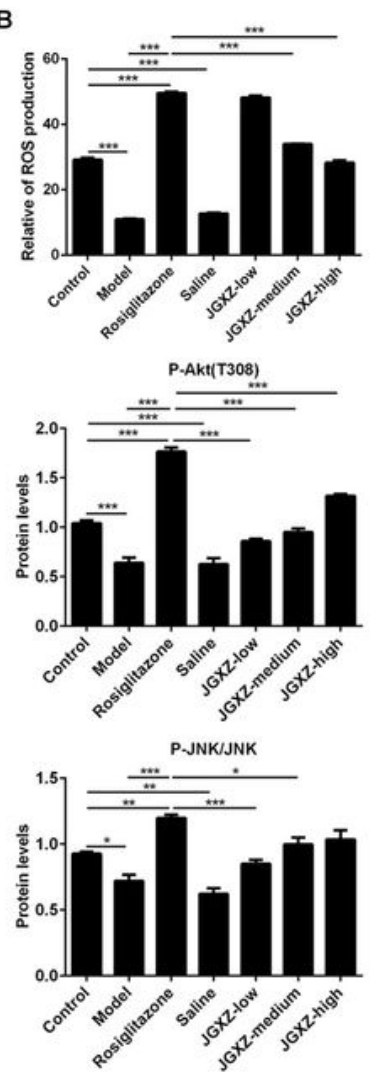
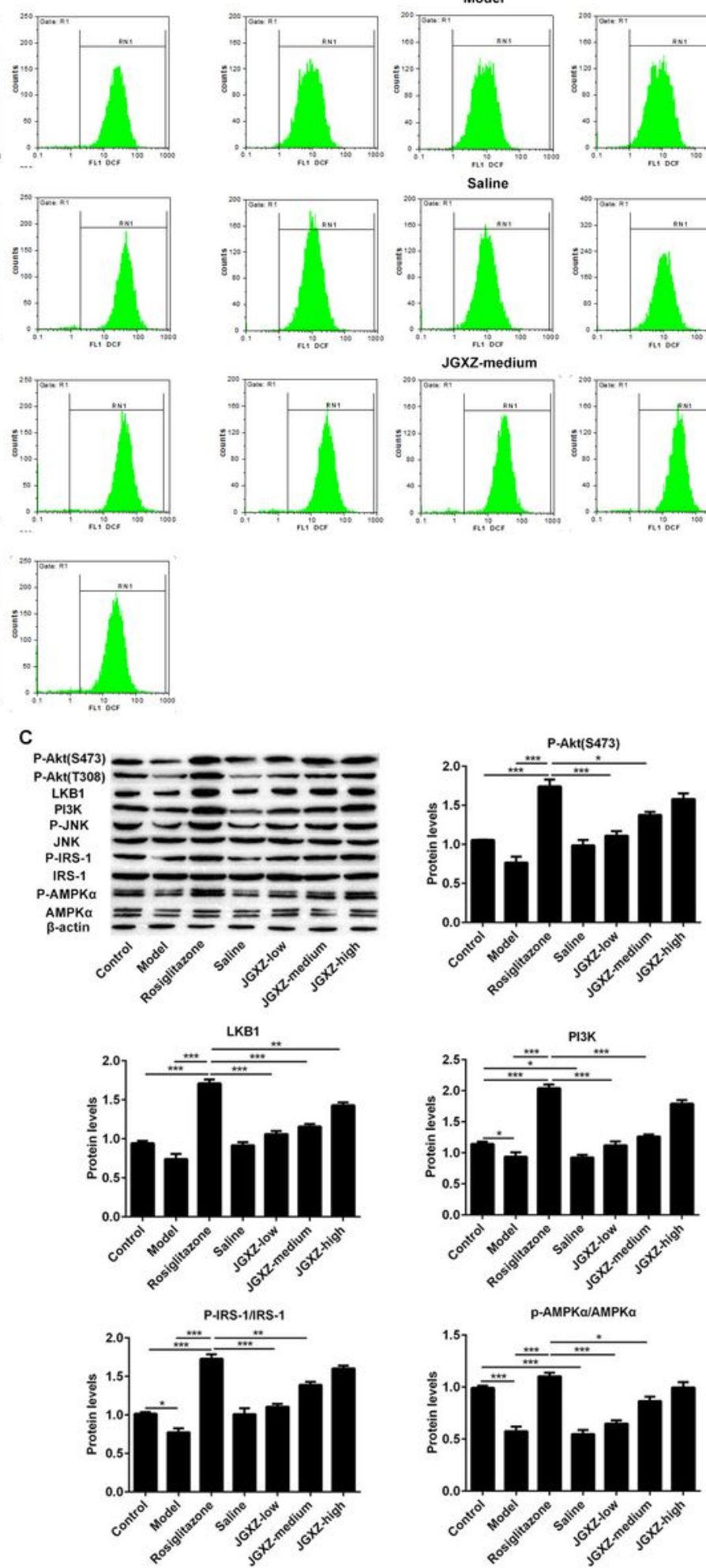

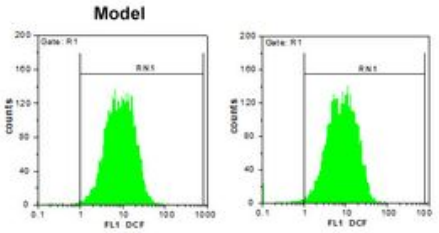

Saline
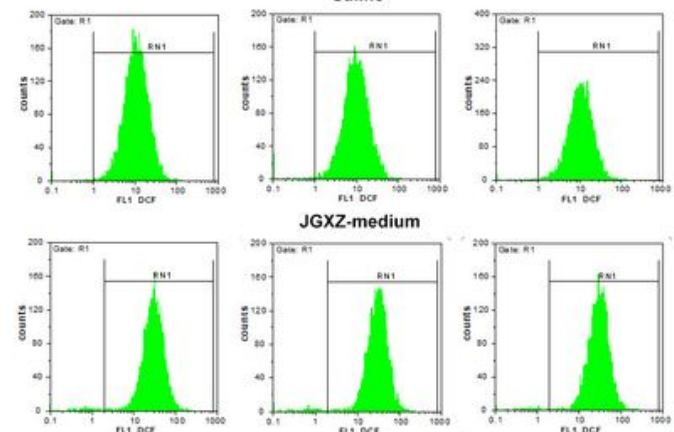
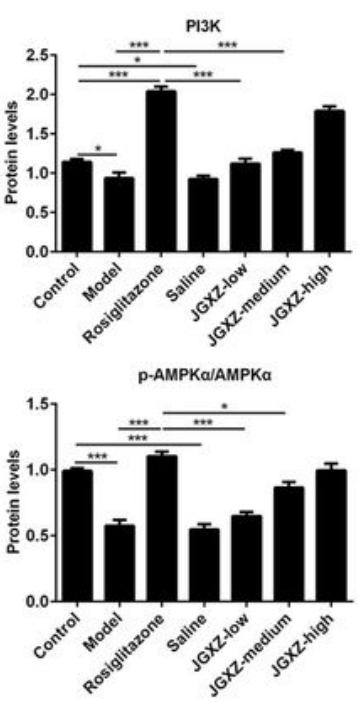

Figure 3

A-B ROS levels were detected by flow cytometry after adipocytes were treated with serum containing drug. $C$ the effects of serum containing drug on LKB1/AMPK and JNK/IRS/PI3k/AKT levels for IR adipocytes. ${ }^{*} \mathrm{p}<0.05$ 
A

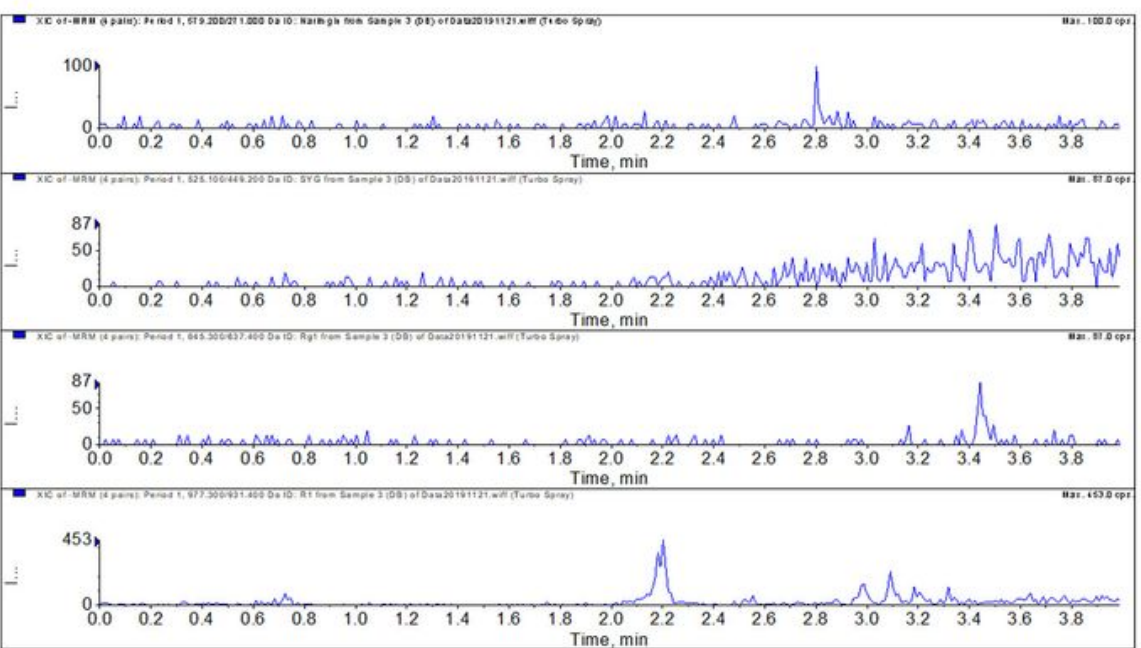

B

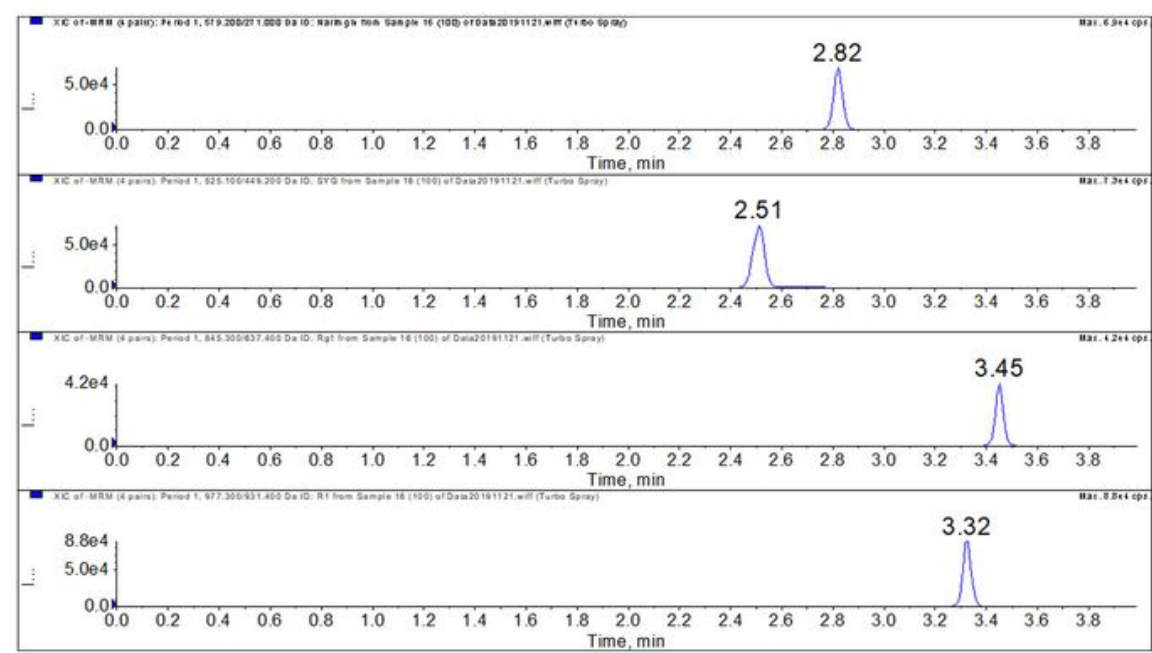

C

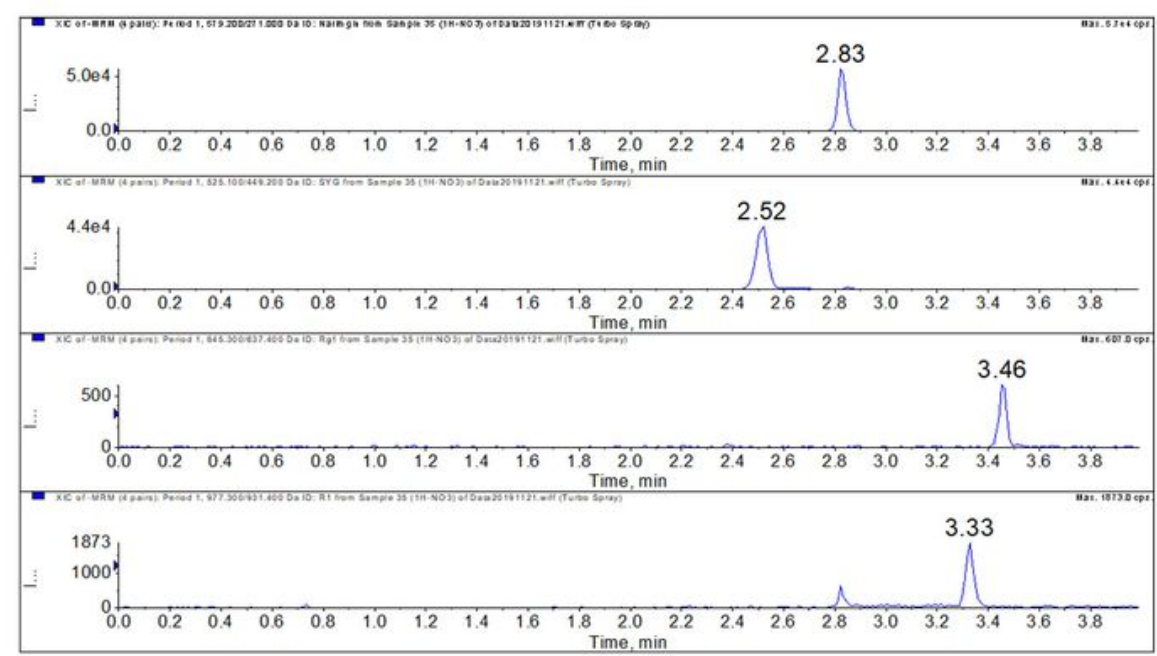

Naringin

Paeoniflorin

Rg1

R1

Naringin

Paeoniflorin

Rg1

R1

Naringin

Paeoniflorin

Rg1

R1

\section{Figure 4}

A Representative LC-MS negative ion chromatograms of blank plasma; B lank plasma spiked with nine compounds $(\mathrm{ng} / \mathrm{ml})$ and $\mathrm{C}$ a rat plasma sample after oral administration of JGXF. 
A
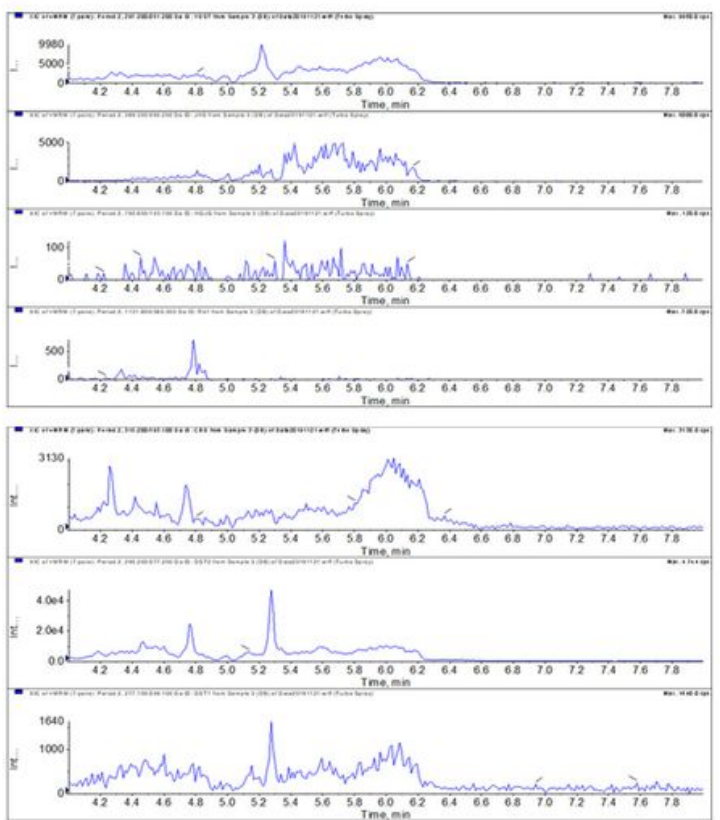

C
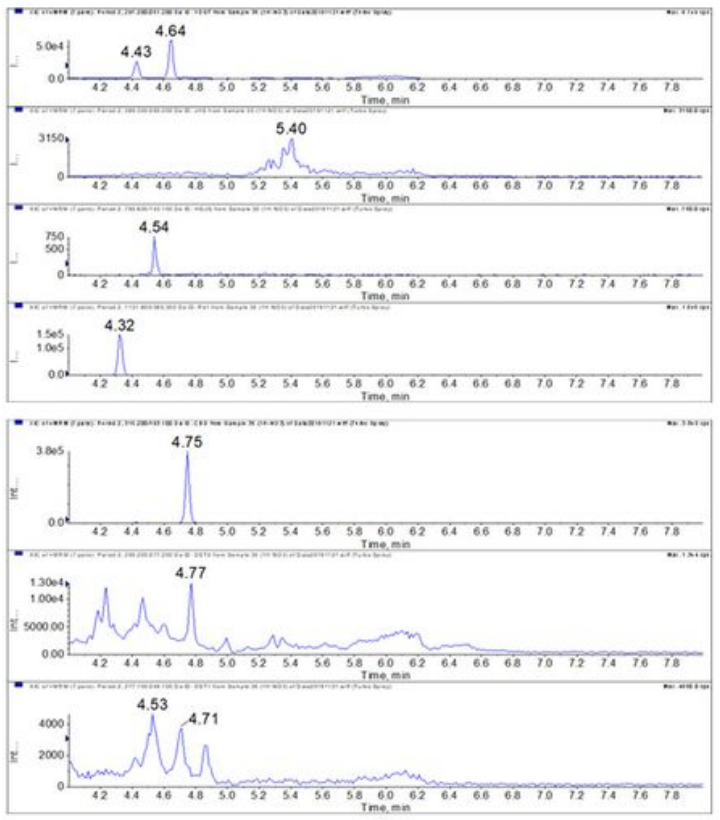

B

Cryptotanshinone

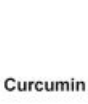

Curcumin

Astragaloside

Rb1

Cannabidiol

Tanshinone II A

Tanshinone ।

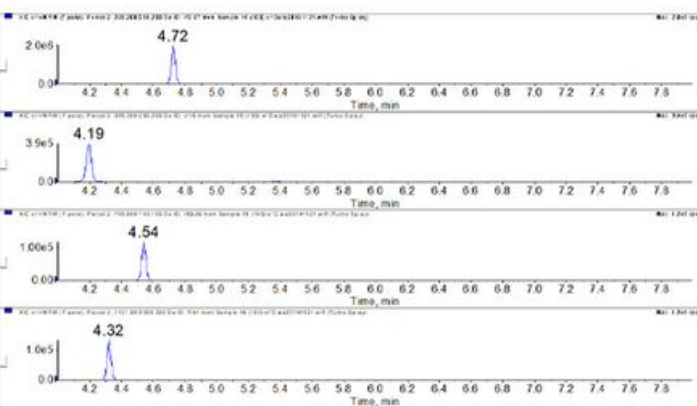

Cryptotanshinone

Curcumin

Astragaloside

$\mathrm{Rb} 1$

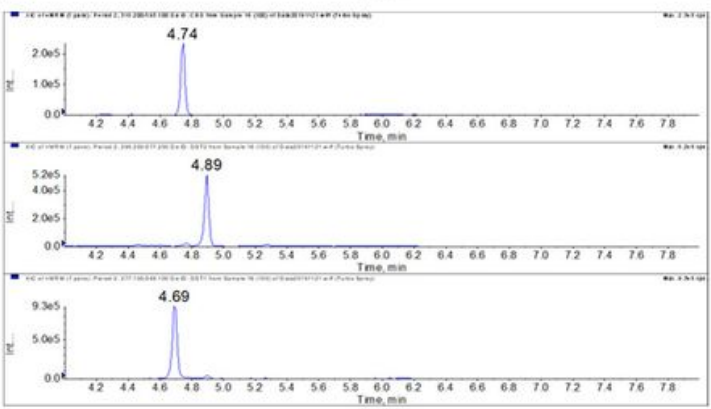

Cannabidiol

Tanshinone II A

Tanshinone ।

Cryptotanshinone

Curcumin

Astragaloside

Rb1

Cannabidiol

Tanshinone II A

Tanshinone I

\section{Figure 5}

A Representative LC-MS positive ion chromatograms of blank plasma; B blank plasma spiked with nine compounds $(\mathrm{ng} / \mathrm{ml})$ and $\mathrm{C}$ a rat plasma sample after oral administration of JGXF. 